\title{
EVALUACIÓN DE LA HIGIENE BUCAL EN LOS PACIENTES DE UN HOSPITAL DE MEDIA/LARGA ESTANCIA
}

ORAL HYGIENE EVALUATION AMONG PATIENTS IN MEDIUM/LONG-TERM STAY HOSPITALS

MOLES JULIO, PILAR

ARNAU MARCO, ESMERALDA

Hospital La Magdalena de Castellón

C/ La Vía, 1-20

12539 Alquerias Niño Perdido (Castellón)

E-mail contacto: pilar_moles@hotmail.com 


\section{RESUMEN}

\section{Titulo:}

Evaluación de la higiene bucal en los pacientes de un hospital de media/larga estancia.

\section{Objetivo:}

Conocer los hábitos higiénicos en el colectivo de pacientes durante su estancia en el Hospital de la Magdalena de Castellón e identificar patologías causadas por posibles hábitos higiénicos bucodentales inadecuados.

\section{Metodología:}

Se trata de un estudio descriptivo transversal observacional basado en datos de registro e información de pacientes y cuidadores principales. Se selecciono una muestra de 50 pacientes en el Hospital de la Magdalena de Castellón hospitalizados. Se realizó un examen odontológico a partir de datos recogidos en un registro diseñado para tal fin. Se usó el índice de LÖE y SILNESS y el índice VIGILD M. Para evaluar la satisfacción se utiliza el test GOHAI. El análisis de los datos se realiza con el programa estadístico R, en el cual saldrán los porcentajes de las patologías BD y hábitos de higiene diarios de los pacientes hospitalizados.

\section{Resultados:}

La edad media de los pacientes estudiados es de 68 años; la mayoría hombres (70\%). Observamos en nuestro estudio que las mujeres no fuman y que sus hábitos higiénicos, patologías encontradas son mejores que en los hombres. Sin embargo un $100 \%$ de los pacientes estudiados necesitan atención bucodental. 


\section{Conclusiones:}

En este estudio se observan problemas de higiene bucodental en los pacientes hospitalizados en un centro de larga estancia de Castellón.

\section{Palabras clave:}

Higiene bucodental, patologías bucodentales, prótesis dental, placa dental, pacientes.

\section{ABSTRACT}

\section{Title:}

Oral hygiene evaluation among patients in medium/long-term stay hospitals.

\section{Objective:}

To know the hygienic habits of patients during their stay in the hospital La Magdalena of Castelló and identify diseases caused by possible inadequate oral hygiene habits.

\section{Methods:}

This is an observational cross-sectional descriptive study based on the data retrieved and information from patients' records and caregivers' reports. A sample of 50 patients was selected at the hospital La Magdalena of Castellón. Inclusion criterion: to be hospitalized. Exclusion criterion: patients who do not accept to participate in the study, and do not sign the informed consent. Dental examination was carried out using the data collected in a registry designed for that purpose. Indexes LÖE, SILNESS and VIGIL M. were 
used. To evaluate patients' satisfaction, the GOHAI test was used. The data analysis was conducted using the statistical program $\mathrm{R}$, which will lead to the percentage of oral pathologies and daily hygiene of hospitalized patients.

\section{Results:}

The mean age of patients studied is 68 years, most of them male $(70 \%)$. We observed in the study that in the variable gender, $30 \%$ are female, nonsmokers, with better hygiene habits and oral diseases found are lower than in male. $100 \%$ of the sample needed oral health care.

\section{Conclusions:}

In this study the lack of oral hygiene in hospitalized patients in a longstay center in Castellón is confirmed.

\section{Key words:}

Oral hygiene, oral pathology, dental prostheses, dental plaque, patients.

\section{ANTECEDENTES Y ESTADO ACTUAL DEL TEMA}

Durante la primera mitad del siglo XX los sistemas sanitarios basaban su actividad en el tratamiento de las enfermedades una vez éstas ya estaban instauradas. Posteriormente se pasó de la visión intervencionista de la salud a una orientación más preventiva, a la vez que han cambiado las pirámides de población. La esperanza media de vida ha aumentado en los últimos 30 años, aumentando el sector de población formado por mayores de 65 años. Las mejoras sociosanitarias entre otros determinantes, han permitido este 
aumento de la esperanza de vida (1). Pero las personas más mayores ya no demandan únicamente vivir más, sino que quieren vivir mejor:"No sólo debemos añadir años a la vida sino dar vida a los años" (2).

La calidad de vida tiene varias dimensiones que incluyen los cuidados independientes para realizar las actividades básicas de la vida diaria. La salud oral es importante para la calidad de vida, llegando a ser un problema por si misma su deterioro (3-4).

La patología oral en personas mayores es diversa y especifica. Enfermedades como la xerostomia, periodontitis y halitosis, son las más frecuente en edades avanzada de la vida. La administración de varios medicamentos de forma simultánea, se asocian a las causas de aparición de algunas patologías orales.

Algunas sintomatologías dentales no tratadas causan dolor e incomodidad, interfiriendo en la capacidad de comer y tragar, dando como resultado una nutrición inadecuada (6). Las infecciones orales, que quizás sean problemas menores en personas jóvenes, pueden causar serias infecciones sistémicas en las personas mayores (7) y en las últimas décadas el porcentaje de ancianos dentados ha aumentado, incrementándose también el riesgo de caries y enfermedad periodontal (8).

La salud bucodental contribuye al bienestar general y, por otra parte, refleja la historia del paciente, en forma de experiencias adaptativas. Estas experiencias cambian en función del sexo, edad, país de origen y en especial 
por variables socioeconómicas, como el nivel de formación, los ingresos económicos y la ocupación que el paciente ha tenido durante su vida laboral $(1-5)$.

Poseer un estado de salud oral correcto no implica indefectiblemente tener 32 dientes en perfecto estado. La salud oral observa tres vertientes: la funcional, la estética y fonética.

La forma más sencilla de mantener un equilibrio ecológico en la cavidad oral es mediante unas correctas normas de higiene oral. El cepillado dental es el mecanismo más sencillo y más efectivo para evitar que la placa dental crezca en exceso (9-10).

Sin embargo, la realización de la higiene bucal puede verse comprometida cuando el individuo presenta incapacidad funcional. Esta condición se asocia en gran medida a la presencia de algunas enfermedades como la parálisis cerebral, deficiencias o problemas médicos, y se define por la dificultad o imposibilidad de llevar a cabo ciertas actividades de la vida diaria, entre ellas la higiene de la boca (11).

Los pacientes hospitalizados son, en su mayoría, incapaces de realizar su cuidado personal. Siempre que sea posible se deben realizar modificaciones y adaptaciones en las técnicas y en los instrumentos de la higiene bucal para que ellos mismos hagan tales procedimientos. Sin embargo, en algunos caso esto es complejo (12). En estas situaciones, los cuidados de la higiene bucal son responsabilidad de los servicios de enfermería, familiares y cuidadores que 
deben poseer conocimientos y habilidades para atender las necesidades de los pacientes hospitalizados, cuando éstos estén incapacitados para realizarla por sí mismos (13). Sin embargo, es frecuente encontrarnos con un índice muy bajo de higiene bucodental en los pacientes hospitalizados en las instituciones de larga estancia (14-15).

Las principales enfermedades que afectan la salud bucodental en los hospitales de media/larga estancia: gingivitis, caries, periodontitis, xerostomía, candidiasis, sensibilidad dental.

La gingivitis es un proceso inflamatorio de las encías causado por la placa bacteriana o por la medicación, siendo un proceso reversible (16).

La caries es una enfermedad multifactorial que se caracteriza por la destrucción de los tejidos del diente como consecuencia de la desmineralización provocada por los ácidos que genera la placa bacteriana (17).

La periodontitis es una enfermedad crónica e irreversible. Afecta al tejido de sostén de los dientes que son las encías, el hueso alveolar, el cemento radicular y el ligamento periodontal.

En los dientes se forman bolsas que atrapan la placa dental y partículas 
de la comida dañando la fibra que sostiene los dientes (16).

La xerostomía es la manifestación clínica más común de las disfunciones salivales y consiste en una disminución de la secreción salival, denominada también boca seca, asialorrea o hiposalivación (18). Junfin y col. (19) la definen como una condición clínica subjetiva donde hay menos cantidad de saliva de lo normal, sin tener una distinción clara entre límites normales y anormales.

Aunque parece que existen más de 500 medicamentos que se relacionan con la xerostomía como efecto secundario, en los que más frecuentemente se ha demostrado una disminución directa en la función salival incluyen los antidepresivos triciclicos, los antihistamínicos, los antidepresivos y los diuréticos (20-21).

La sensibilidad dental es un síndrome doloroso transitorio derivado de la exposición de la dentina (parte interna del diente) a estímulos externos de origen térmico, químico o táctil y que no pueden ser asociados a ninguna otra patología dental (16).

El bruxismo es una anomalía de los hábitos masticatorios caracterizada por rechinar y apretar los dientes de forma excesiva. Esta anomalía es destructiva para las piezas dentales, generándose grandes desgastes de las piezas dentales y dolor orofacial (22). 
Ante una situación de bruxismo crónico, la articulación temporamandibular (ATM) se puede llegar a ver muy afectada y comprometida (22).

Otras lesiones, como el afta son úlceras o llagas abiertas y dolorosas en la boca. Las aftas son de color blanco o amarillo y están rodeadas por un área roja y brillante. No son cancerosas.

Las aftas se pueden desencadenar por: Estrés emocional, falta de ciertas vitaminas y minerales en la alimentación (en especial de hierro, ácido fólico o vitamina B12), Períodos menstruales, Cambios hormonales, Alergias a los alimentos.

Cualquier persona puede desarrollar aftas. Las mujeres son más propensas a contraerlas que los hombres. Las aftas pueden ser hereditarias (23).

En el caso de placa bacteriana o dental, las bacterias que se encuentran en la cavidad oral pueden estar organizadas de dos maneras: por una parte las que se encuentran en la saliva suspendidas en la fase liquida, adoptando una forma que se denomina plactónica (flotan suspendidas en un medio liquido); 0 bien, las bacterias que se encuentran sobre una superficie dura (diente, prótesis e implantes) formando una película gelatinosa adherente: la placa dental. La placa dental es el principal agente etiológico de las caries y de las enfermedades periodontales. 
Las micosis ocupan un papel destacado y aunque existen diversos tipos de micosis bucales las más comunes son las Candidiasis, producidas por un hongo Ilamado "Candida Albicans "que forma colonias de color blanco en la lengua o paladar, pueden cursar dolor especialmente al comer (24).

Las prótesis constituyen una alternativa para la pérdida de la dentadura. Pero, aun cuando son una buena solución, no están exentas de provocar daños, lo cual, junto a la susceptibilidad de los tejidos propiciados por el envejecimiento y por los inadecuados estilos de vida, puede llevar a cambios importantes de las mucosas, los huesos de la cavidad bucal y, en especial, la aparición de lesiones que pueden variar desde las más simples hasta las malignas y causar un gran problema de salud para las personas y para la sociedad (25-26).

Las lesiones más frecuentes relacionadas con las prótesis son:

Candidiasis pseudomenbranosa ( $L, P)$; Candidiasis hiperplasica, asintomática (mucosa bucal y zona retromolar); Candidiasis eritematosa, puede aparecer en cualquier parte de la boca, molestias, ardor, comidas, color más oscuro que las otras; Quelitis angular, comisuras de los labios; Estomatitis subprótesis: se define como un proceso inflamatorio de la mucosa oral relacionado con una prótesis removible; Épulis fisurado: es una hiperplasia de la mucosa que resulta de un trauma de bajo grado crónico inducido por un reborde de la prótesis; Hiperqueratosis: es el resultado de una alteración de la queratina de los tejidos ante una agresión por un agente externo (prótesis). 
Un síntoma muy generalizado es la Halitosis. Se define como el conjunto de olores desagradables u ofensivos que manan de la cavidad bucal (27).

Entre las causas que contribuyen a la Halitosis oral se encuentran: Biofil lingual, Enfermedad Periodontal, Ulceraciones mucosas, Impactación alimentaría, prótesis fijas mal ajustadas, prótesis removibles mal higienizadas, flujo salival disminuido.

Si consideramos que la salud bucal es un componente de la salud, y que la salud tiene entre sus componentes la calidad de vida, entonces la salud bucal también tendrá un efecto en la calida de vida. Así como ha habido una evolución en los conceptos de "Salud", reconociendo que tiene un amplio rango de componentes (biológicos, mentales, sociales y de calidad de vida) (28); también ha evolucionado el concepto de salud bucal, cambiando desde el nivel biológico, en que la cavidad bucal contribuye con la protección del cuerpo por medio de la masticación y deglución; hasta los niveles social y psicológico, donde contribuye con la autoestima, expresión, comunicación y estética facial (29). La salud bucal también se ha definido como "dentición funcional y confortable que permite a los individuos continuar con el rol social deseado" (29), enfatizando que es un componente integral de la salud (30), por lo que se entiende que cuando la salud bucal está comprometida, la salud general y la calidad de vida pueden disminuir (29). Entonces la salud bucal se define no solo clínicamente, sino agregando aspectos como el impacto del dolor bucal en la vida diaria de los individuos, y el grado de inhabilidad o "mal función" de esta (31). 
Se presenta un estudio relacionado con la salud bucodental en pacientes hospitalizados en el Hospital La Magdalena perteneciente al grupo hacles, (hospital atención crónicos larga estancia), con un total de 130 camas distribuidas en 24 camas de larga estancia; 18 camas de cuidados paliativos; 60 camas de convalecientes y 28 camas de daño cerebral.

\section{METODOLOGÍA}

Se trata de un estudio descriptivo transversal observacional basado en datos de registro, observación e información de pacientes y cuidadores principales.

La población a estudio son personas hospitalizadas en régimen de larga estancia en el Hospital La Magdalena durante el año 2012 con una estancia media de 90 días, diagnosticadas de problemas respiratorios y accidentes cerebro-vasculares (ACV) con edades comprendidas entre 32-89 años. El estudio se realizó durante tres semanas.

Criterios de inclusión: Estar hospitalizado entre dos y tres meses desde el ingreso. Con patologías respiratorias y ACV estabilizadas.

Criterios de exclusión: Pacientes que por su estado avanzado de enfermedad (paliativos y terminales) no se puede realizar un seguimiento y pacientes que no acepten entrar en el estudio y no firmen el consentimiento informado. 
Del total 130 pacientes hospitalizados, la dirección de enfermería del hospital selecciona aleatoriamente una muestra de 50 pacientes. El cálculo del tamaño muestral para 130 pacientes para que fuera significativo con un nivel de confianza de un $95 \%$ y un margen de error de $5 \%$ es de 98 pacientes, pero solo se me facilitan 50 pacientes por lo cual esto es una limitación del estudio.

Para la recogida de datos, se realiza una exploración bucodental de acuerdo a procedimiento consensuado entre la enfermera que realiza la exploración y la Higienista Dental. Se emplearon barreras de protección, espejo dental plano, sonda periodental recomendada por la OMS y pinzas (todo el material desechable).

Para la recogida de información relacionada con los pacientes se aplico el test de GOHAI, el índice de LÖE y SILNESS y el índice VIGILD M.

El estudio se realiza para todos los pacientes por la misma observadora.

Las variables dependientes: son el nivel de higiene bucal y la presencia de prótesis de los pacientes.

Las variables independientes: son edad, sexo, patología bucodental, hábitos higiénicos y hábito tabáquico.

Para conocer la autopercepción de los problemas bucales, se aplico el 
GOHAI (anexo). Una vez obtenido el total de registros, se consideraron las categorías: no necesita atención bucodental (puntuación alta >57puntos) y necesita atención bucodental (puntuación baja $=57$ ), según lo recomendado en la validación del instrumento para este tipo de población.

El método utilizado para el índice de la placa dental es el de Silness, no necesitando la aplicación de sustancias descubridoras, las piezas dentarias seleccionadas representativas de toda la boca son $(1.6,2.1,2.4,3.6,4.1,4.4)$ en cuatro sitios por diente, mesial, vestibular distal y palatino.

El índice utilizado para la higiene de prótesis dentales es el de Vigild M, con la clasificación: buena, aceptable, deficiente.

Se realiza por la misma observadora, realizando un volcado de la información recogida a una base de datos Excel diseñada para el estudio durante el periodo de tiempo previsto.

Para la entrevista, se prioriza la información que aporta el paciente. Si tiene limitaciones en la comunicación, la información la aporta el/la cuidador/a principal.

Los datos de los cuestionarios se vuelcan en una hoja de cálculo de Excel para así poder pasarlos al programa estadístico. El análisis de los datos se realiza mediante el programa estadístico R. Para el análisis de los datos 
cualitativos utilizamos las tablas de contingencia para dos variables para poder sacar los porcentajes y Pearson's Chi-squared de homogeneidad e independencia. Para el análisis de los datos cuantitativas utilizamos resúmenes numéricos, para poder sacar la media; todo ello con un intervalo de confianza de un $95 \%$.

El tamaño de la muestra es el que nos proporciona la dirección de enfermería del hospital, quien ha seleccionado aleatoriamente una muestra de 50 pacientes, por lo cual los datos que nos facilitan no sabemos de donde los obtienen ni el tiempo de ingreso del paciente por enfermedad o defunción.

Se informa verbalmente a los pacientes o representantes legales el tipo de estudio y la finalidad del estudio. Posteriormente se pasa el consentimiento informado que firma el paciente o representante legal.

Teniendo en conocimiento la Ley Orgánica 15/1999, de 13 de diciembre, de Protección de Datos de Carácter Personal, se archivaran los datos en el Hospital de la Magdalena de Castellón

\section{RESULTADOS}

La muestra la integran un total de 50 pacientes. El 30\% fueron mujeres y $70 \%$ hombres. La edad media de los pacientes que participan en el estudio 68.24 años con una desviación típica de 15.19 
De los resultados obtenidos del cuestionario de hábitos de higiene bucodental en el hospital se obtiene:

Que hay hábitos perjudiciales para la cavidad oral.

Como se observa en la tabla 1 existe diferencia entre hombres y mujeres estudiados sobre el hábito tabáquico. Como podemos apreciar en la tabla.

\begin{tabular}{|l|c|c|c|}
\hline $\begin{array}{l}\text { Hábitos } \\
\text { tabáquicos }\end{array}$ & $\begin{array}{c}\% \\
\text { hombres }\end{array}$ & $\begin{array}{c}\% \\
\text { mujeres }\end{array}$ & p-value \\
\hline Fumador & 14 & 0 & $3.752 \mathrm{e}-07$. \\
\hline No fumador & 12 & 30 & $3.752 \mathrm{e}-07$. \\
\hline Exfumador & 44 & 0 & $3.752 \mathrm{e}-07$. \\
\hline
\end{tabular}

Tabla 1. Hábitos tabáquicos

En cuanto a los hábitos higiénicos son mejores en las mujeres que en los hombres, independientemente de la edad y tanto si son portadores de prótesis o no.

En la tabla 2 queda reflejado el número de cepillados por día en relación al sexo.

\begin{tabular}{|l|c|c|c|}
\hline No de Cepillados al día: & $\begin{array}{c}\% \\
\text { hombres }\end{array}$ & $\begin{array}{c}\% \\
\text { mujeres }\end{array}$ & p-value \\
\hline Tres & 8 & 8 & 0.6842 \\
\hline Dos & 18 & 4 & 0.6842 \\
\hline Uno & 24 & 8 & 0.6842 \\
\hline Alguna & 4 & 2 & 0.6842 \\
\hline semanal & 2 & 0 & 0.6842 \\
\hline ninguna & 14 & 8 & 0.6842 \\
\hline Lengua & 18 & 6 & 0.6646 \\
\hline
\end{tabular}

Tabla 2. Hábitos higiénicos bucodentales 
La utilización del cepillo manual respecto al eléctrico, se estudia, encontrando las diferencias observadas en la tabla 3. Al estar hospitalizados de utiliza más el cepillo manual que el eléctrico en ambos sexos y por mayor comodidad.

\begin{tabular}{|l|c|c|c|}
\hline Tipo de cepillo & $\begin{array}{c}\% \\
\text { hombres }\end{array}$ & $\begin{array}{c}\% \\
\text { mujeres }\end{array}$ & p-value \\
\hline manual & 53,2 & 21,3 & 0.5204 \\
\hline eléctrico & 6,4 & 0 & 0.5204 \\
\hline ninguno & 12,8 & 6,4 & 0.5204 \\
\hline interproximal & 8 & 0 & 0.3041 \\
\hline Seda dental & 4 & 0 & 0.5046 \\
\hline
\end{tabular}

Tabla 3. linstrumental mecánico de higiene bucodental

Podemos apreciar en la tabla 4 que el porcentaje pacientes que utilizan los enjuagues bucales es superior al $66 \%$.

\begin{tabular}{|l|c|c|c|}
\hline $\begin{array}{l}\text { Producto } \\
\text { químico }\end{array}$ & $\begin{array}{c}\% \\
\text { hombres }\end{array}$ & $\begin{array}{c}\% \\
\text { mujeres }\end{array}$ & p-value \\
\hline enjuagues & 44 & 22 & 0.4736 \\
\hline
\end{tabular}

Tabla 4. Productos químicos bucodentales

Resultado de patologías bucodentales encontradas.

Se observamos en la figura 1 que el porcentaje más alto de patología encontrada entre los pacientes del hospital está relacionada con sus hábitos higiénicos bucodentales. 


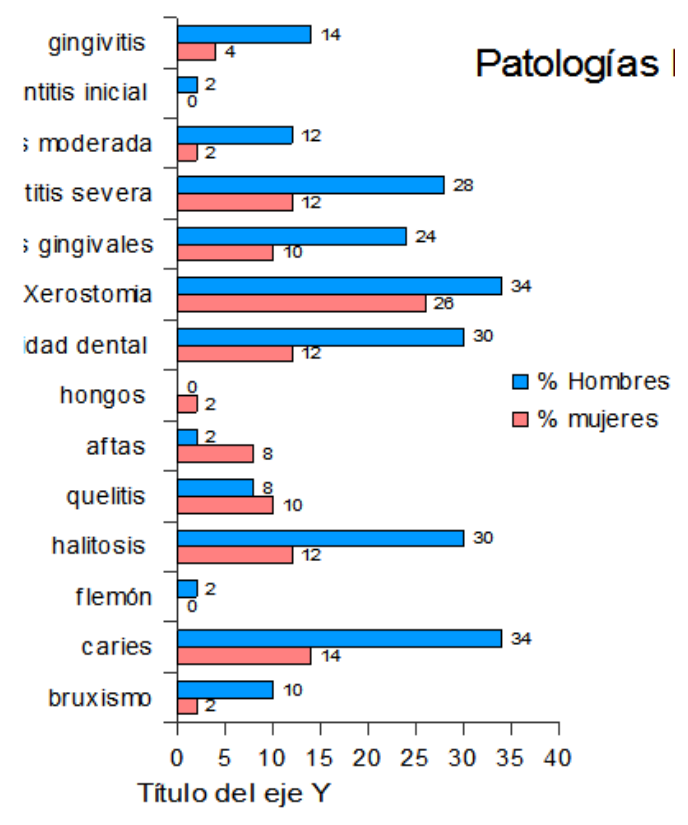

Figura 1. Porcentaje de Patologías Bucodentales

De acuerdo al criterio propuesto por Vigild M para la valoración de higiene de prótesis dental, de un total de 50 pacientes estudiados 25 son portadores de prótesis removibles, de los cuales 17 son hombres y 8 mujeres.

\begin{tabular}{|l|c|c|}
\hline $\begin{array}{l}\text { Higiene prótesis } \\
\text { dental }\end{array}$ & Hombre & Mujer \\
\hline Buena & 2 & 1 \\
\hline Aceptable & 5 & 2 \\
\hline Deficiente & 10 & 5 \\
\hline
\end{tabular}

Tabla 5. Higiene de prótesis dentales removibles

En cuanto al resultado de la higiene de la prótesis tabla 5 encontramos $2 \%$ irritación en hombres y $0 \%$ mujeres tanto en el maxilar superior e inferior. También a causa de la mala higiene y desajuste de la prótesis observamos un $2 \%$ de épulis fisurado en hombres y $0 \%$ en mujeres. 
Criterios clínicos para el índice de placa dental de Löe y Silness:

Según este índice queda reflejado figura 2 que los hombres tienen peores resultados de higiene que las mujeres.

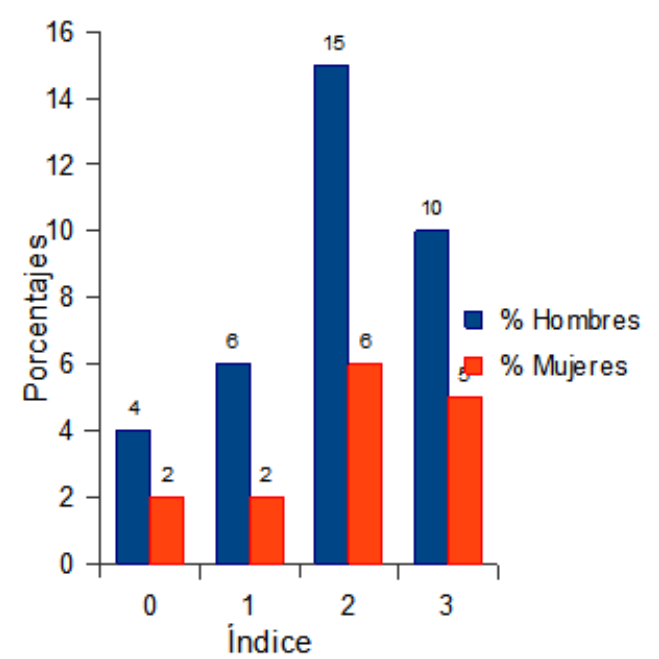

Figura 2. Índice de Placa Dental de Löe y Silness

El resultado del test de Gohai tabla 6 indica que 35 hombres y 15 mujeres muestra recogida necesitan atención bucodental, es decir, el $100 \%$ de pacientes estudiados.

De acuerdo a los resultados del test de Gohai, todos los pacientes estudiados necesitan higiene bucodental.

\begin{tabular}{|l|c|c|c|}
\hline Sexo & $\begin{array}{c}\text { No necesita } \\
\text { atención } \\
\text { bucodental }\end{array}$ & $\begin{array}{c}\text { Necesita } \\
\text { Atención } \\
\text { ucodental }\end{array}$ & p-value \\
\hline Hombre & $0 \%$ & $70 \%$ & 0.0046 \\
\hline Mujer & $0 \%$ & $30 \%$ & 0.0046 \\
\hline
\end{tabular}

Tabla 6. Índice GOHAI 


\section{DISCUSIÓN}

En este estudio se comprueba la higiene bucodental en pacientes de media/larga estancia con problemas crónicos, considerando que la boca es la puerta de entrada de diversas patologías.

Los resultados encontrados en este estudio referente a la variable sexo o portadores de prótesis dentales removibles, presenta una diferencia significativa en los hábitos de higiene bucodental y tabáquicos por sexos como sucede en estudios sobre hábitos de higiene oral en la población escolar y adulta española (17). Al analizar los resultados se deben tener presente algunas limitaciones del estudio. Sin embargo la carencia de bibliografía detectada sobre hospitalización de media-larga estancia .pone de manifiesto, la necesidad de realizar estudios de estas características, para identificar problemas de hospitalización relacionados no solo con la calidad de vida sino, con la seguridad de pacientes (36).

Debido a que la percepción de la salud es diferente entre hombres y mujeres, el escaso número de pacientes puede haber influido en los resultados. Las percepciones de salud por sexos se observan en el estudio de Casals-Peidró (17).

El resultado del $100 \%$ de la muestra que necesita atención bucodental puede estar condicionado por el tamaño muestral.

Los pacientes al ingreso tienen un índice bajo de cepillado, pero se observa que utilizan más el cepillo manual que el eléctrico ya que les resulta más cómodo y rápido, con un porcentaje considerable de pacientes manifiestan 
que se les olvida el cepillado. En cambio si son muy utilizados los productos químicos (enjuagues bucales) como sustitución del cepillado.

Este estudio, ha permitido establecer grados de intensidad de acumulo de placa con la aplicación del índice de Löe-Silness. El índice se ha utilizado en estudios similares como Oral hygiene habits in spanish school children and adults (17). El porcentaje de placa dental ha sido mayor en los hombres que en las mujeres igual que sucede con otras patologías. Este acumulo se debe a que los pacientes cuando hacen un ingreso hospitalario, preocupados por su enfermedad, la higiene bucodental pasa a ser casi inexistente, sobre todo en pacientes dependientes (32).

El número de pacientes portadores de prótesis dentales removibles es mayor en hombres que en mujeres. Obteniendo un resultado de higiene deficitaria en ambos por norma general no se quitan las prótesis dentales para higienizarlas, siendo habitual encontrar prótesis removibles mal ajustadas por lo que hay más retención de restos alimentarios y a causa de estos desajustes se producen irritaciones y épulis fisurado. No se conoce la causa de que no se quiten las prótesis aunque se puede atribuir a mismo motivo que al olvido del cepillado, de acuerdo a la información que se obtiene en las entrevistas.

Entre los pacientes del hospital de Magdalena en las exploraciones de higiene bucodental se encontraron diversas patologías. Las más relacionadas con la mala higiene bucodental son periodontitis, gingivitis, caries y halitosis 
como sucede en pacientes de centros institucionalizados (32). Pudiendo mejorar dichas patologías con una adecuada higiene bucodental.

Una de las patologías más desagradable socialmente es la halitosis, que en un alto porcentaje puede desaparecer o mejorar con una buena higiene bucodental. Además la halitosis esta directamente relacionada con la caries, la gingivitis y la periodontitis por la acumulación del biofilm oral u otras patologías como pueden ser del área otorrinolaringológica, medicamentosa y hábitos alimenticios. En algunos casos el paciente no es consciente de ella.

En la muestra del estudio la gingivitis siendo un proceso inflamatorio reversible no es muy significativa. De lo que se puede deducir que la deficitaria higiene de los pacientes es de un proceso largo de tiempo durante su estancia.

En la muestra de pacientes revisados el índice de caries es superior en un $20 \%$ en hombres que en mujeres, lo que sigue demostrando que hay más mala higiene bucodental en hombres (17).

De los 50 pacientes revisados exceptuando los portadores de prótesis dentales removible, todos padecen periodontitis en alguna de sus tres fases: inicial, moderada, severa. La periodontitis es frecuente en pacientes con una higiene bucodental deficitaria (16). 
Una de las patologías con más prevalencia en la muestra de pacientes de este hospital es la Xerostomia. Los factores que contribuyen a su aparición son la edad, portadores de prótesis y la medicación. Siendo una de las patologías más molestas para la calidad de vida en los pacientes, para la mejora de esta patología durante el ingreso, es recomendar una buena higiene oral con pasta y enjuagues bucales específicos. De acuerdo a las recomendaciones de los profesionales (33).

La calidad de vida va asociada con la salud bucodental, y esta relacionada con el impacto que las alteraciones bucales pueden producir en la vida diaria de los pacientes (33) como:

La hipersensibilidad dental tiene un impacto directo sobre la calidad de vida de los pacientes, llegando a modificar hábitos alimentarios y hábitos de higiene.

La reducción o ausencia de secreción salivar (xerostomia) en la cavidad bucal puede provocar diversas manifestaciones clínicas que hacen que disminuya la calidad de vida del paciente. La xerostomia dificulta funciones orales como la masticación, deglución y la fonación entre otras.

Una de las quejas que más demanda el paciente es la sensibilidad dental, que al ser un síndrome doloroso a los estímulos externos de origen químico y táctil, también les afecta en la calidad de vida tal como se muestra en los artículos (33). 
Es difícil la comparación con otros estudios al encontrar poca bibliografía relacionada con este estudio que valore la relación de la higiene bucodental en pacientes hospitalizados de larga estancia.

Los estudios encontrados se refieren a patologías de la boca por separado (3516-17) y centros Geriátricos institucionalizados (32).

\section{CONCLUSIONES}

Hay problemas de higiene bucodental. Los profesionales, familiares y cuidadores deben tomar conciencia del problema, ya que a veces la higiene bucodental pasa a un segundo plano por su enfermedad. En este estudio queda demostrada la falta de higiene bucodental en los pacientes hospitalizados en un centro de larga estancia de Castellón, por lo que parece recomendable ampliar el estudio para observar posibles diferencias con otros centros de idénticas características. 


\section{REFERENCIAS}

1- Lamster IB, Northridge ME, editores. Improving oral health for the elderly. An interdisciplinary approach. Nueva York: Springer Science+Business Media, LLC; 2008.

2- Salvà-Casanovas A, Llevadot D, Miró M, Vilalta M, Rovira JC. La atención geriátrica. Uno de los grandes ejes de la atención sociosanitaria en Cataluña. Rev Esp Geriatr Gerontol. 2004; 39(2): 101-8.

3- Grupo Español de investigación en Gerodontología.Oral Health Spanish Issues. Int DEnt J. 2001; 51: 228-34.

4- Bravo Pérez M, Casals Peidró E, Cortés Martinicorena FJ, et al. Encuesta de salud oral en España 2005. RCOE. 2006; 11: 409-56.

5- Gil-Montoya JA, Subirá C, Ramón JM, González-Moles MA. Oral healthrelated quality of life and nutricional status. J Public Health Dent. 2008; 68: 88-93.

6-Ritchie Cs, Burgio KL, Locher JL, Cornwell A, Thomas D, Hardin M, Reeden D. Nutritional status of urban homebound older adults. Am J Clin Nut. 1997; (4): 815-18.

7- Kaiser-Jones J, Bird WF, Paul SM, Long L, Schell ES. An instrument to assess the oral health status of nursing home residents. Gerontologist. 1995; 35(6): 814-24.

8- Ettinger RL. The unique oral heatlth needs of an aging population. Dent Clin North Am. 1997; 41(4): 633-49.

9- Ettinger RL. Meeting oral health needs to promote the well-being of the 
geriatric population: educational research issues. J DEnt Educ. 2010; 74(1): 29-35.

10- Papas AS, Niessen LC, Chauncey HH. Geriatric dentistry. Aging and oral health. St Louis: Mosby; 1991.

11-Rosa TEC, Benicio MHA, Latorre MRDO, Ramos LR. Factores determinantes da capacidade funcional entre idosos. Rev. Saúde Pública. 2003; 37(1): 40-48.

12- Christensen J, Fiske J, Domiciliarycare for the elderly patient. En: Barnes IE, Walls A. Gerontology. London: Wright; 1994.p.169-199.

13- Fitzpatrick J. Oral health care needs of dependent older people: Responsibilities of nurses and care staff. J Avd Nurs 2000; 32(6): 1325-1332.

14-Berkley DB. Improving dental access for the nursing home resident: Portable dentistry interventions. Gerodontics. 1987; 3(6): 265-268.

15- Shay K. Oral neglect in the institutionalized elderly part 2: The role of the dentist and the standard of care. Spec Care Dentist. 1990; 10(6): 200-203.

16- Sociedad Española de Periodoncia y Osteointegración. Manual de Higiene Bucal. Ed. Médica Panamericana, 2009; 154 páginas.

17- Casals-Peidró Elías. Hábitos de higiene oral en la población escolar y adulta española. RCOE [revista en la Internet]. 2005 Ago [citado 2012 Jun 08]; 10(4): 389-401. Disponible en: http://scielo.isciii.es/scielo.php? script $=$ sci arttext\&pid=S1138-123X2005000400002\&lng=es. http://dx.doi.org/10.4321/S1138-123X2005000400002.

18- Levine M. Development of artificial salivas. Oral Biol Oral Med. 4: 279-286. 1993. 
19- Junfin G, Van Dis M, Langlais R, Miles D. Xerostomia: diagnosis and treatment planning considerations. Oral Surg. 58: 248-252. 1984.

20- Bascones A, Tenovuo J, Ship J, Turner M, Mac-Veigh I, López-Ibor JM, et al. Conclusiones del Simposium 2007 de la Sociedad Española de Medicina Oral sobre "Xerostomía. Síndrome de Boca Seca. Boca Ardiente". Av odontoestomatol. 2007; 23(3): 119-26.

21- Marques-Soares MS, Chimenos-Kustner E, Subirá-Pifarrè C, Rodriguez De Rivera-Campillo ME, López-López J. Association of burning mouth syndrome with xerostomia and medicines. Med Oral Patol Oral Cir Bucal. 2005; 10: 3018.

22- Frugone Zambra RE, Rodríguez C. Bruxismo. Av Odontoestomatol [revista en Internet]. 2003 Jun [citado 2012 Jun 06]; 19(3): 123-130. Disponible en: http://scielo.isciii.es/scielo.php?script=sci_arttext\&pid=S0213-

$12852003000300003 \&$ lng $=$ es. http://dx.doi.org/10.4321/S021312852003000300003.

23- Vázquez Doval FJ, Gómez Bezares P. Publicado en FMC. Form Med Contin Aten Prim. 1999; 6(8): 557-

24- Rodríguez Ortega J, Miranda Tarragó J, Morejón Lugones H, Santana Garay JC. Candidiasis de la mucosa bucal: Revisión bibliográfica. Rev Cubana Estomatol [revista en Internet]. 2002 Ago [citado 2012 Jun 06]; 39(2): 187233. Disponible en: http://scielo.sld.cu/scielo.php? script=sci_arttext\&pid=S0034-75072002000200007\&lng=es.

25- Díaz Guzmán LM, Gay Zárate O. Series en medicina bucal X. Odontología de calidad para los pacientes ancianos. Revista Asociación Dental Mexicana 
(ADM). 2005; LXII(1): 36-9.

26- Bernal Baláez AE. Estudio cariométrico de la mucosa bucal en un modelo de carcinogénesis química. Rev Federación Odontológica Colombiana. 1998; 55(192):

27-Roldan, S. Conceptos básicos sobre la halitisis.Ars XXI de comunicación, DL; 2006.

28- Zini A, Sgan-Cohen HD. The effect of Oral Health on Quality of Life in an underprivileged homebound and non-homebound elderly population in Jerusalem. J Am Geriatr Soc. 2008; 56(1): 99-104.

29- Kushnir D, Shlomo P, Zusman SP, Robinson PG. Validation of a Hebrew version of the Oral Health Impact Profile 14. J Public Health Dent. 2004; 64(2): 71-5.

30- Locker D, Allen F. What do measures of "oral health-related quality of life" measure? Community Dent Oral Epidemiol. 2007; 35(6): 401-411.

31- Soe KK, Gelbier S, Robinson PG. Reliability and validity of two oral health related quality of life measures in Myanmar adolescents. Community Dent Health $2004 ; 64(2): 71-5$.

32- Conceição Ferreira R, Salles Rocha E, Nogueira Coutinho N, de Freitas Ribeiro MT, Silami de Magalhães C, Nogueira Moreira A. La actuación del equipo de enfermería en la higiene bucal de los ancianos dependientes hospitalizados. Invest. educ. enferm [revista eb Internet]. 2006 Mar [citado 2012 Jun 06; 24(1): 48-57. Disponible en: http://www.scielo.org.co/scielo.php? script=sci_arttext\&pid=S0120-53072006000100005\&lng $=$ en . 
33- Dentaid (ed.). Expertise. Cerdanyola (España): Dentaid. 2012 MayoAgosto; 9.

34- Pinzon SA, Gil JA. Validación del Geriatric Oral Health Assessment Index en población geriátrica institucionalizada en Granada. (Observación no publicada: enviada a la revista Española de Geriatria y Gerontología. Enero 1999).

35- Luis Delgado O, Echeverría García J], Berini Aytés L, Gay Escoda C. La periodontitis como factor de riesgo en los pacientes con cardiopatía isquémica. Med. oral patol. oral cir. bucal (Ed.impr.) [revista en Internet]. 2004 Abr [citado 2012 Jun 06]; 9(2): 125-137. Disponible en: http://scielo.isciii.es/scielo.php?script=sci_arttext\&pid=S169844472004000200005\&lng=es.

36-Gálvez González M. Guía de actuación: Higiene del paciente crítico. Biblioteca Lascasas, 2008; 4(4). Disponible en http://www.indexf.com/lascasas/documentos/lc0363.php 